\title{
TOURISM ENVIRONMENTAL IMPACT ASSESSMENT BASED ON IMPROVED AHP AND PICTURE FUZZY PROMETHEE II METHODS
}

\author{
Chao TIAN ${ }^{2}$, Juan-juan PENG ${ }^{*}$, Wen-yu ZHANG ${ }^{1}$, \\ Shuai $\mathrm{ZHANG}^{1}$, Jian-qiang $\mathrm{WANG}^{3}$ \\ ${ }^{1}$ School of Information, Zhejiang University of Finance \& Economics, Hangzhou 310018, China \\ ${ }^{2}$ School of Accounting, Zhejiang University of Finance \& Economics, Hangzhou 310018, China \\ ${ }^{3}$ School of Business, Central South University, Changsha 410083, China
}

Received 18 March 2019; accepted 06 October 2019

\begin{abstract}
With the increasing awareness of environmental protection, the environmental impact assessment (EIA) of tourism attraction problems has attracted extensive attention from all over the world. The purpose of this study is to develop an integrated decision-making method for EIA of tourism attractions in China by using the improved analytic hierarchy process (AHP) method and the extended picture fuzzy preference ranking organization method for enrichment evaluations (PROMETHEE) II method. Herein, picture fuzzy numbers (PFNs) are used to describe decisionmakers' preference, which can precisely express uncertain and hesitant information in the decisionmaking process. Then the improved AHP method based on expert mean assessment is used to obtain the weights of EIA of tourism attractions, which can reduce the spiteful assessment in the traditional AHP method. Based on the results of the improved AHP method, the picture fuzzy PROMETHEE II method, which can consider the psychological behavior of decision-makers, is applied to assess candidate tourism attractions. Finally, an example of EIA of tourism attractions in China is provided to verify the effectiveness of the developed approach and the credibility of the results.
\end{abstract}

Keywords: EIA of tourism attractions, tourism development in China, AHP, PROMETHEE II, picture fuzzy numbers.

JEL Classification: D81, C61, Q65.

\section{Introduction}

Environmental impact assessment (EIA) was firstly defined in the National Environmental Policy Act promulgated in the United States in 1969 and was introduced into China in the 1970s (Geneletti \& Dawa, 2009; Chang, Wang, Wu, Sun, \& Hu, 2018). With the increasing awareness of environmental protection, and for the purpose of sustainable tourism management, how to balance the tourism development and the over-exploitation of resources has

${ }^{\star}$ Corresponding author. E-mail: pengjj81@csu.edu.cn

This is an Open Access article distributed under the terms of the Creative Commons Attribution License (http://creativecommons. org/licenses/by/4.0/), which permits unrestricted use, distribution, and reproduction in any medium, provided the original author and source are credited. 
attracted close attention from researchers and practitioners in China (Zhong, Deng, Song, \& Ding, 2011). EIA of tourism attractions is one of the important issues that need to address for sustainable tourism management (Canteiro, Córdova-Tapia, \& Brazeiro, 2018; Su, Wall, Wang, \& Jin, 2019). During EIA, the natural ecological environment impact, social cultural impact, and economic impact should be taken into account simultaneously, which can be regarded as a classical multi-criteria decision-making (MCDM) problem (Zhao \& Zhang, 2017). However, because of the uncertainty of information, decision-makers cannot accurately describe their preferences using the existing theories. Therefore, the picture fuzzy numbers (PFNs), the improved analytic hierarchy process (AHP), and the preference ranking organization method for enrichment evaluations (PROMETHEE) II are extended into the proposed MCDM method to handle EIA problems.

Recently, EIA of tourism attractions has been widely studied by many scholars. For example, Zhao and Zhang (2017) developed an intuitionistic MCDM method to assess the environmental impact of tourism on the Desert Park; Tseng et al. (2018) assessed the sustainability of tourism development in Vietnam based on a hierarchical structure approach and the fuzzy set theory; Cowburna, Moritza, Birrella, Grimsditcha, and Abdulla (2018) assessed the environmental impact of resort tourism from an environmental sustainability perspective. However, these existing methods do not fully consider the uncertainty of decision-making information and the psychological factors of decision-makers as well.

Thus, the motivation of this study can be summarized as follows. First, EIA of tourism attractions always involves uncertain or imprecise information in the decision-making process. Especially, if decision-makers refuse to assess some criteria for EIA of tourism attractions because of their knowledge limitation, this assessment information cannot be recorded by existing methods (Zhao \& Zhang, 2017; Tseng et al., 2018; Cowburna et al., 2018). Instead, PFNs can precisely express decision-makers' preferences, including yes, abstention, no, and refusal, and avoid any information loss for assessment purposes, thus making the obtained data more consistent with an actual decision-making environment. Second, the improved AHP method based on expert mean assessment can reduce the errors caused by the subjectivity of decision-makers in the traditional AHP method, and the PROMETHEE II can consider the psychological behavior of decision-makers. Thus, based on the improved AHP method, we extend the PROMETHEE II method to a picture fuzzy environment for EIA of tourism attractions in China, in which the improved AHP method is utilized to obtain preference weights, and the picture fuzzy PROMETHEE II method is used to assess and rank tourism attractions.

Finally, the remainder of this study is organized as follows. In Section 1, some background knowledge is reviewed. Section 2 introduces the PFSs, the improved AHP method and the PROMETHEE II method. Section 3 develops an integrated decision-making method combining the improved AHP and picture fuzzy PROMETHEE II for EIA of tourism attractions. Section 4 provides a case study, the results and analysis, Section 5 gives findings and discussion, and Section 6 presents some implications. The last section provides conclusions.

\section{Background knowledge}

The criteria and the methods for EIA of tourism attractions are reviewed in the following. 


\subsection{Criteria for EIA of tourism attractions}

Recently, a number of scholars have studied the criteria index system related to the EIA of tourism attractions in China (Ye \& Liu, 2000; Li, Zhang, \& Wang, 2005; Li, Xue, Ren, Zhang, \& Wang, 2015; Su et al., 2019). For instance, Ye and Liu (2000) investigated ecotourism and tourist indicator systems of eco-environmental quality in China; Yin and Han (2009) introduced the content, means and assessment criteria of EIA in tourism attractions, and provided some suggestions for ecological environmental construction in China; Li et al. (2015) discussed the impacts of tourism activities on the ecological environment, and constructed an assessment index system based on a fuzzy method; Su et al. (2019) discussed the livelihood sustainability of tourism attractions in China.

Based on experts' opinions, and appropriate literature reviews (Luo, 2002; Ye \& Liu, 2000; Gossling et al., 2005; Duan, Su, Xu, \& Ran, 2008; Zhao \& Zhang, 2017; Tseng et al., 2018; Elvan, 2018; Greco, Cenciarelli, \& Allegrini, 2018), it can be concluded that the main factors that should be considered in EIA of tourism attractions include social, ecological and economic factors. On this basis, it is reasonable to divide the indicators for the environmental impact of tourism attractions into three levels, i.e., natural ecological environmental impact, social cultural environmental impact, and economic environmental impact. Natural ecological environmental impact can be further subdivided into three sub-criteria of tourism environmental impact, ecological environmental impact, and ecological characteristic impact; social cultural environmental impact can be subdivided into three sub-criteria of material cultural impact, behavior cultural impact, and spiritual cultural impact; economic environmental impact can be subdivided into two sub-criteria of economic system impact and power system impact. Each sub-criterion has multiple tertiary indicators. More details about these criteria of the environmental impact can be found in Table 1.

\subsection{Methods for EIA of tourism attractions}

After decades of development, EIA has become an important tool for the sustainable development of environment and been widely used in various fields (Michailidou, Vlachokostas, Moussiopoulos, \& Maleka, 2016; Thanvisitthpon, 2016; Chang et al., 2018; Khosravi, Jha-Thakur, \& Fischer, 2019; Ocampo-Melgara, Sagarisb, \& Gironás, 2019). Especially, the methods for EIA of tourism attractions have been studied by some scholars. For instance, Li et al. (2005) discussed key technologies and methods that were used to assess the environmental influences of ecological tourism activities; Duan et al. (2008) used the remote sensing (RS) technology to establish a method to evaluate the eco-environmental quality of eco-agricultural tourism; MacNeill and Wozniak (2018) discussed the social, economic, and environmental impact of cruise tourism; Canteiro et al. (2018) discussed the environmental impact of tourism activities in nature reserves, and considered tourism impact assessment as a useful tool to realize a sustainable tourism management. Nonetheless, the research on the methods of EIA of tourism attractions in China and other countries is still inadequate, and existing methods cannot fully consider the uncertainty or fuzziness in the decision-making process as well. 
Table 1. The criteria index system for EIA of tourism attractions in China

\begin{tabular}{|c|c|c|}
\hline Criteria & Sub-criteria & Remarks \\
\hline \multirow{3}{*}{$\begin{array}{l}\text { (C1) Natural } \\
\text { ecological } \\
\text { environmental } \\
\text { impact }\end{array}$} & $\begin{array}{l}\text { (C11) Tourism } \\
\text { environmental impact }\end{array}$ & $\begin{array}{l}\text { Landscape diversity, ecosystem carrying capacity, tourism } \\
\text { resource characteristics, and landscape reputation }\end{array}$ \\
\hline & $\begin{array}{l}\text { (C12) Ecological } \\
\text { environmental impact }\end{array}$ & $\begin{array}{l}\text { Air quality, water quality, soil quality, garbage disposal } \\
\text { capacity, and noise control ability }\end{array}$ \\
\hline & $\begin{array}{l}\text { (C13) Ecological } \\
\text { characteristics impact }\end{array}$ & $\begin{array}{l}\text { Diversity of biological resources, plant richness, greening } \\
\text { level of tourism attractions }\end{array}$ \\
\hline \multirow{3}{*}{$\begin{array}{l}\text { (C2) Social } \\
\text { cultural } \\
\text { environmental } \\
\text { impact }\end{array}$} & $\begin{array}{l}\text { (C21) Material cultural } \\
\text { impact }\end{array}$ & $\begin{array}{l}\text { Traffic environmental conditions, public health, } \\
\text { infrastructure, and commercial development }\end{array}$ \\
\hline & $\begin{array}{l}\text { (C22) Behavior cultural } \\
\text { impact }\end{array}$ & $\begin{array}{l}\text { Shopping intentions, entertainment tendencies, lifestyle, } \\
\text { and eating habits }\end{array}$ \\
\hline & $\begin{array}{l}\text { (C23)Spiritual cultural } \\
\text { impact }\end{array}$ & $\begin{array}{l}\text { Social security situation, traditional customs, religious } \\
\text { beliefs, and moral norms }\end{array}$ \\
\hline \multirow{2}{*}{$\begin{array}{l}\text { (C3) Economic } \\
\text { environmental } \\
\text { impact }\end{array}$} & $\begin{array}{l}\text { (C31) Economic system } \\
\text { impact }\end{array}$ & $\begin{array}{l}\text { Economic development level, urbanization level, } \\
\text { and tertiary industry }\end{array}$ \\
\hline & $\begin{array}{l}\text { (C32) Power system } \\
\text { impact }\end{array}$ & $\begin{array}{l}\text { Consumption level of tourism attractions, status of } \\
\text { industries involved in residence, transportation and food }\end{array}$ \\
\hline
\end{tabular}

\section{Preliminaries}

\subsection{PFSs}

Picture fuzzy sets (PFSs), initially defined by Cuong and Kreinovich (2013), are extensions of intuitionistic fuzzy sets (IFSs) (Atanassov, 1986; P. Liu \& W. Liu, 2018; Zhuang, Su, \& Chang, 2019). PFSs include four parameters, namely, positive, neutral, negative, and refusal membership degrees respectively. PFSs can describe decision-makers' preferences precisely, since they involve all possible assessment information including yes, abstention, no, and refusal. It can avoid any information loss from the assessment process, and thus makes the data correspond better to an actual decision-making environment than IFSs (Wei, 2016, 2017; L. Wang, Peng, \& J. Q. Wang, 2018a; L. Wang, Zhang, J. Q. Wang, \& Wu, 2019a; R. Wang, J. Wang, Gao, \& Wei, 2019b; Yang, Hu, Liu, \& Chen, 2019; Tian, Peng, S. Zhang, W. Y. Zhang, \& Wang, 2019; L. Wang, X. K. Wang, Peng, \& J. Q. Wang, 2020).

Definition 1 (Cuong \& Kreinovich, 2013). A PFS $\psi$ in the universe of discourse $X$ can be expressed by:

$$
\psi=\left\{\left\langle x, \mu_{\psi}(x), \eta_{\psi}(x), v_{\psi}(x)\right\rangle \mid x \in X\right\},
$$

where $\mu_{\psi}(x) \in[0,1], \eta_{\psi}(x) \in[0,1]$ and $v_{\psi}(x) \in[0,1]$ are the positive, neutral and negative membership respectively, and satisfy the condition $0 \leq \mu_{\psi}(x)+\eta_{\psi}(x)+v_{\psi}(x) \leq 1, \forall x \in X$. $\pi_{\psi}(x)=1-\left(\mu_{\psi}(x)+\eta_{\psi}(x)+v_{\psi}(x)\right)$ indicates the refusal degree of $x$ in $\psi$. If there is only one element in $\psi$, then $\psi$ is a picture fuzzy number (PFN) and denoted as $\psi=\left(\mu_{\psi}, \eta_{\psi}, v_{\psi}\right)$ for convenience. Moreover, the complement of a PFN $\psi^{c}$ is defined as $\psi^{c}=\left(v_{\psi}, \eta_{\psi}, \mu_{\psi}\right)$.

Definition 2. Assume $\psi_{1}$ and $\psi_{2}$ are two PFNs, then the corresponding comparison method is given by: 
(1) If $S\left(\psi_{1}\right)>S\left(\psi_{2}\right)$ or

$S\left(\psi_{1}\right)=S\left(\psi_{2}\right)$ and $A\left(\psi_{1}\right)>A\left(\psi_{2}\right)$, then $\psi_{1} \succ \psi_{2} ;$

(2) If $S\left(\psi_{1}\right)=S\left(\psi_{2}\right)$ and $A\left(\psi_{1}\right)=A\left(\psi_{2}\right)$, then $\psi_{1}=\psi_{2}$.

Here $S\left(\psi_{i}\right)=\frac{1}{3}\left(\mu_{\psi_{i}}+1-\eta_{\psi_{i}}+1-v_{\psi_{i}}\right)(i=1,2)$ and $A\left(\psi_{i}\right)=\frac{1}{3}\left(\eta_{i}-v_{i}\right)(i=1,2)$.

Definition 3. Assume $\psi_{1}$ and $\psi_{2}$ are two PFNs, and then the picture fuzzy distance measure of $\psi_{1}$ and $\psi_{2}$ is given as:

$$
\begin{aligned}
D_{p}\left(\psi_{1}, \psi_{2}\right) & =\left(\frac { 1 } { 5 } \left(\left|\mu_{\psi_{1}}-\mu_{\psi_{2}}\right|^{\lambda}+\left|v_{\psi_{1}}-v_{\psi_{2}}\right|^{\lambda}+\left|\eta_{\psi_{1}}-\eta_{\psi_{2}}\right|^{\lambda}+\mid \max \left\{\mu_{\psi_{1}}, \eta_{\psi_{2}}\right\}\right.\right. \\
& \left.\left.-\left.\max \left\{\mu_{\psi_{2}}, \eta_{\psi_{1}}\right\}\right|^{\lambda}+\left|\max \left\{\mu_{\psi_{1}}, \nu_{\psi_{2}}\right\}-\max \left\{\mu_{\psi_{2}}, v_{\psi_{1}}\right\}\right|^{\lambda}\right)\right)^{1 / \lambda}(\lambda \geq 1) .
\end{aligned}
$$

(1) If $\lambda=1$, then the picture fuzzy distance measure, i.e., Eq. (2), reduces to the picture fuzzy Hausdorff distance measure as:

$$
\begin{aligned}
D_{p h}\left(\psi_{1}, \psi_{2}\right) & =\frac{1}{5}\left(\left|\mu_{\psi_{1}}-\mu_{\psi_{2}}\right|+\left|v_{\psi_{1}}-v_{\psi_{2}}\right|+\left|\eta_{\psi_{1}}-\eta_{\psi_{2}}\right|+\mid \max \left\{\mu_{\psi_{1}}, \eta_{\psi_{2}}\right\}\right. \\
& \left.-\max \left\{\mu_{\psi_{2}}, \eta_{\psi_{1}}\right\}+\left|\max \left\{\mu_{\psi_{1}}, v_{\psi_{2}}\right\}-\max \left\{\mu_{\psi_{2}}, v_{\psi_{1}}\right\}\right|\right) .
\end{aligned}
$$

(2) If $\lambda=2$, then the picture fuzzy distance measure, i.e., (2) reduces to the picture fuzzy Euclidean distance measure as:

$$
\begin{aligned}
D_{p e}\left(\psi_{1}, \psi_{2}\right) & =\left(\frac { 1 } { 5 } \left(\left|\mu_{\psi_{1}}-\mu_{\psi_{2}}\right|^{2}+\left|v_{\psi_{1}}-v_{\psi_{2}}\right|^{2}+\left|\eta_{\psi_{1}}-\eta_{\psi_{2}}\right|^{2}+\mid \max \left\{\mu_{\psi_{1}}, \eta_{\psi_{2}}\right\}\right.\right. \\
& \left.\left.-\left.\max \left\{\mu_{\psi_{2}}, \eta_{\psi_{1}}\right\}\right|^{2}+\left|\max \left\{\mu_{\psi_{1}}, v_{\psi_{2}}\right\}-\max \left\{\mu_{\psi_{2}}, v_{\psi_{1}}\right\}\right|^{2}\right)\right)^{1 / 2} .
\end{aligned}
$$

\subsection{The improved AHP method}

AHP is a comprehensive decision-making method that fully considers of qualitative and quantitative attributes (Saaty, 1988, 1990). It has been successfully applied in different fields, such as environmental science and technology (Ayag \& Ozdemir, 2009; Emrouznejad \& Marra, 2017; Cegan, Filion, Keisler, \& Linkov, 2017; Çalik, Yapici Pehlivan, \& Kahraman, 2018; Gunduz \& Alfar, 2019), and made desirable achievements in MCDM problems.

For AHP, the analytical hierarchical structure should be constructed using a goal layer, criteria layer, sub-criteria layer, and alternatives layer firstly. Then the relative importance values on a fuzzy linguistic scale of 1 and 9 are assigned considering the uncertainty of decision-makers' qualitative assessment. Here, 1 represents exactly equal important and 9 represents certainly high important. More details about the linguistic scales are presented in Table 2. Furthermore, a reciprocal value is determined for inverse comparison, i.e., $\alpha_{i i}=1 / \alpha_{i i}$, where $\alpha_{i i}$ represents the importance of the $i$-th element relative to the $j$-th element. Thus, the pairwise comparisons matrix between alternatives can be constructed as: 
where $\alpha_{i i}=1$ and $\alpha_{i j}=1,3,5,7,9$.

$$
\alpha=\left[\begin{array}{cccc}
1 & \alpha_{12} & \cdot & \alpha_{1 n} \\
\alpha_{21} & \cdot & \cdot & \cdot \\
\cdot & \cdot & \cdot & \cdot \\
\alpha_{n 1} & \alpha_{n 2} & \cdot & 1
\end{array}\right],
$$

Table 2. Fuzzy linguistic scales using the improved AHP method

\begin{tabular}{|l|c|}
\hline \multicolumn{1}{|c|}{ Linguistic Term } & Crisp Value \\
\hline Exactly equal important & 1 \\
\hline Average important & 3 \\
\hline High important & 5 \\
\hline Very high important & 7 \\
\hline Certainly high important & 9 \\
\hline Intermediate value & $2,4,6,8$ \\
\hline
\end{tabular}

Decision-makers may exhibit some subjectivity when determining the importance of criteria, which reduces the reliability of weighting of these criteria. Therefore, an expert mean assessment method (see Figure 1) is suggested to improve the traditional AHP method in order to avoid errors caused by human factors (Zhang, Wang, \& Yang, 2018a), i.e., the improved AHP method. First, the experts judge the relative importance of two criteria according to the fuzzy linguistic scales in Table 1. Then, the mean values of all experts' scores for the two criteria, as well as the deviation between any two experts' scores and the mean values of all deviations, are calculated. If all deviations fall within the confidence region, then the mean value represents the final relative importance of the two criteria. However, if the deviation

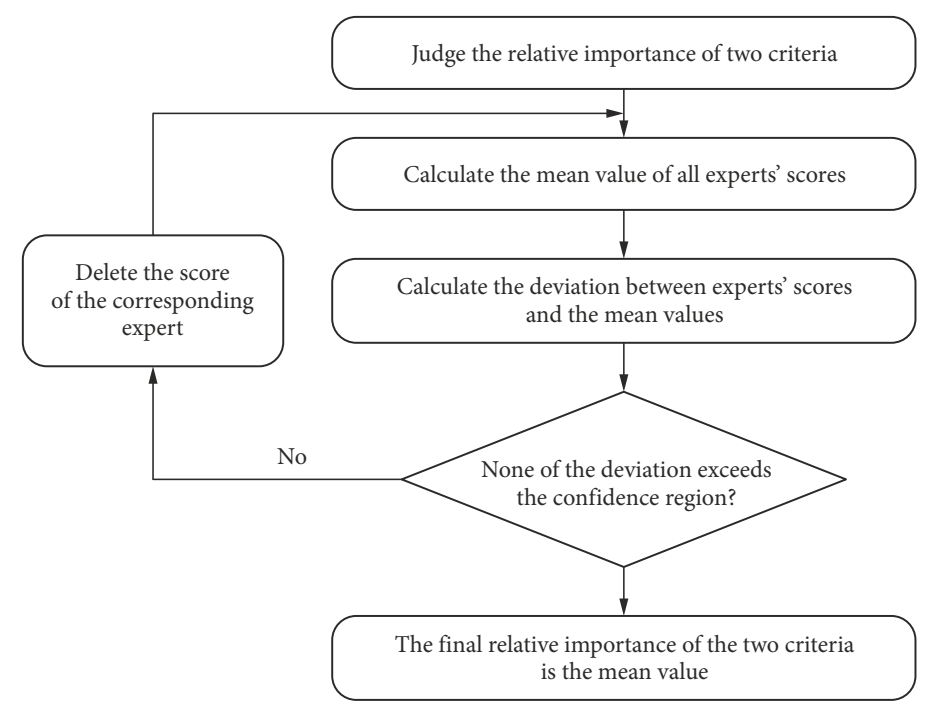

Figure 1. Flowchart of the expert mean assessment method 
exceeds the confidence region, then the score of the corresponding expert must be deleted. In the latter case, the mean values of the remaining experts' scores for the two criteria should be recalculated, and the above described steps are repeated until all deviations fall within the confidence region. The key part of the expert mean assessment method is the determination of the confidence region. Generally speaking, the confidence region can be reduced to the interval of $[-2.3,2.3]$ considering the experts' industry experience in the tourism. Finally, the judgement matrices can be constructed.

After pair-wise comparisons matrices, the consistency index (CI) of each pair-wise matrix should be calculated according to $C I=\left(\varsigma_{\max }-n\right) /(n-1)$, where $\varsigma_{\max }$ indicates the biggest eigenvalue of the judgment matrix, and $n$ denotes the matrix size. Then the consistency ratio (CR) can be obtained as $C R=C I / R I$, where $R I$ denotes the random consistency index and can be found in Table 3. If $C R \leq 0.1$, then the consistency can be satisfied; otherwise, the pair-wise comparison matrices must be revised.

Table 3. The random consistency index (RI) (Saaty, 1990)

\begin{tabular}{|c|c|c|c|c|c|c|c|c|}
\hline Size $(n)$ & 1 & 2 & 3 & 4 & 5 & 6 & 7 & 8 \\
\hline RI & 0 & 0 & 0.58 & 0.9 & 1.12 & 1.24 & 1.32 & 1.41 \\
\hline
\end{tabular}

\subsection{The PROMETHEE II method}

PROMETHEE, developed by Brans (1982), can be used to handle the mapping operation of alternative preferences to precise numbers or ratios when considering different criteria (Kabir \& Sumi, 2014; Sennaroglu \& Celebi, 2018; Salari, Rakhshandehroo, \& Nikoo, 2018). Subsequently, based on the PROMETHEE method, Vincke and Brans (1985) developed an extended method of PROMETHEE II, which is an outranking method for a finite set of alternatives $\psi=\left\{\psi_{1}, \psi_{2}, \ldots, \psi_{n}\right\}$ that need to be ranked and chose among multiple criteria $C=\left\{c_{1}, c_{2}, \ldots, c_{m}\right\}$. The PROMETHEE II method considers the preference function $v_{j}\left(\psi_{i}, \psi_{k}\right)$ between two alternatives $\psi_{i}$ and $\psi_{k}$ under each criterion $c_{j}$ provided by decision-makers, and can choose the suitable preference function among multiple criteria. The larger the function value is, the bigger the difference between alternatives. Especially, if $v_{j}\left(\psi_{i}, \psi_{k}\right)=0$, then $\psi_{i}$ and $\psi_{k}$ are indifferent; while if $v_{j}\left(\psi_{i}, \psi_{k}\right)=1$, then $\psi_{i}$ is strictly preferential to $\psi_{k}$.

Definition 4 (Brans, 1982; Vincke \& Brans, 1985). Let $\varpi_{j}(j=1,2, \ldots, m)$ be the relative weight of criterion $c_{j}(j=1,2, \ldots, m)$, and then the preference index can be determined as:

$$
\rho\left(\psi_{i}, \psi_{k}\right)=\sum_{j=1}^{m} \varpi_{j} \cdot v_{j}\left(\psi_{i}, \psi_{k}\right) .
$$

According to the preference index, the positive flow $\tau^{+}\left(\psi_{i}\right)$ and negative flow $\tau^{-}\left(\psi_{i}\right)$ of each alternative $\psi_{i}$ can be defined respectively as:

$$
\begin{aligned}
& \tau^{+}\left(\psi_{i}\right)=\sum_{k=1}^{n} \rho\left(\psi_{i}, \psi_{k}\right) \\
& \tau^{-}\left(\psi_{i}\right)=\sum_{k=1}^{n} \rho\left(\psi_{k}, \psi_{i}\right) .
\end{aligned}
$$


Then the corresponding net flow $\tau\left(\psi_{i}\right)$ can be calculated as:

$$
\tau\left(\psi_{i}\right)=\tau^{+}\left(\psi_{i}\right)-\tau^{-}\left(\psi_{i}\right) .
$$

Based on the net flow values, the preference relations between two alternatives can be determined. If $\tau\left(\psi_{i}\right)>\tau\left(\psi_{k}\right)$, then alternative $\psi_{i}$ is preferential to alternative $\psi_{k}$; while if $\tau\left(\psi_{i}\right)=\tau\left(\psi_{k}\right)$, then two alternatives $\psi_{i}$ and $\psi_{k}$ are indifferent.

\section{An integrated decision-making approach by using the improved AHP method and the picture fuzzy PROMETHEE II method}

In order to handle the EIA problem of tourism attractions, an integrated decision-making approach by using the improved AHP method and the picture fuzzy PROMETHEE II method is developed to prioritize and rank the tourism attractions.

Assume $\psi=\left\{\psi_{1}, \psi_{2}, \ldots, \psi_{n}\right\}$ is a collection of alternatives, $C=\left\{c_{1}, c_{2}, \ldots, c_{m}\right\}$ is the corresponding criteria, and $R=\left(\psi_{i j}\right)_{n \times m}$ is the picture fuzzy decision matrix. Here, $\psi_{i j}=\left(\mu_{i j}, \eta_{i j}, v_{i j}\right)(i=1,2, \ldots, n ; j=1,2, \ldots, m)$ is the performance of $\psi_{i}$ for criterion $c_{j}$. Then the proposed approach is illustrated in the following.

The integrated decision-making method for EIA of tourism attractions using the improved AHP method and the picture fuzzy PROMETHEE II method can be seen in Figure 2.

\section{Phase I. Construct the criteria for EIA of tourism attractions}

In this phase, the criteria for EIA of tourism attractions are identified and assessed by experts, academicians, and tourism environmental associates via relevant literature reviews, as presented in Figure 2.

Phase I

Criteria selection
Phase II

Improved AHP

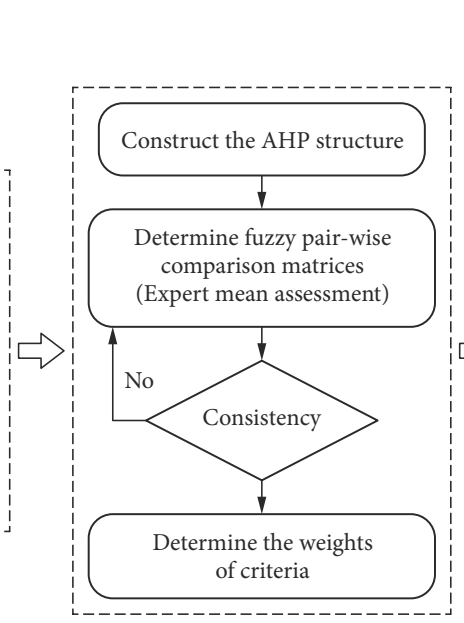

Phase III

Picture fuzzy PROMETHEE II

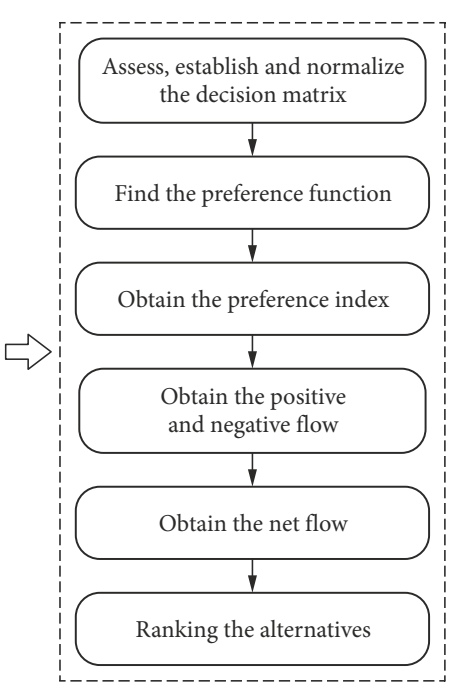

Figure 2. Flowchart of the developed method 


\section{Phase II. Determine the weights of criteria using the improved AHP method}

Step 2.1. Construct the analytical hierarchical structure

Based on Phase I, the analytical hierarchical structure should be constructed using a goal layer, criteria layer, sub-criteria layer, and alternatives layer.

Step 2.2. Determine fuzzy pair-wise comparison matrices

To develop comparison matrices, the experts need to compare the relative importance of two criteria according to the linguistic scales in Table 2. Based on the expert mean assessment method (see Figure 1), the corresponding comparison matrices can be constructed. Moreover, the consistency of each pair-wise comparison of matrix should be examined.

Step 2.3. Determine the weights of criteria

From the pair-wise comparison matrices determined in Step 2.2, the weight vector for criteria can be obtained using Super Decision Software.

\section{Phase III. The extended picture fuzzy PROMETHEE II method}

Step 3.1. Establish the decision matrix

According to decision-makers' knowledge and experience, the decision-makers invited in Phase I should provide assessment values of all criteria for each alternative in the form of PFNs $\psi_{i j}(i=1,2, \ldots, n ; j=1,2, \ldots, m)$. Then, the decision matrix can be obtained.

Step 3.2. Normalize the decision matrix

Since the most common criteria for decision-making problems involve costs and benefits, the assessment value $\psi_{i j}(i=1,2, \ldots, n ; j=1,2, \ldots, m)$ should be normalized to $\tilde{\Psi}_{i j}(i=1,2, \ldots, n ; j=1,2, \ldots, m)$ to unify all criteria. For the benefit criteria, $\tilde{\psi}_{i j}=\psi_{i j}(i=1,2, \ldots, n ; j=1,2, \ldots, m)$, while for the cost criteria, the assessment values should be normalized as follows $\tilde{\psi}_{i j}=\psi_{i j}^{c}(i=1,2, \ldots, n ; j=1,2, \ldots, m)$.

Step 3.3. Find the preference function

From Definition 2, and the picture fuzzy distance $D_{p}\left(\psi_{i j}, \psi_{k j}\right)$ in Definition 3, the corresponding preference function can be expressed as:

$$
\begin{aligned}
v_{j}^{1}\left(\psi_{i}, \psi_{k}\right) & =\left\{\begin{array}{lc}
\left(D_{p}\left(\psi_{i j}, \psi_{k j}\right)\right)^{\alpha}, & \psi_{i j} \succ \psi_{k j} ; \\
0, & \text { otherwise }
\end{array}\right. \\
v_{j}^{2}\left(\psi_{i}, \psi_{k}\right) & =\left\{\begin{array}{lc}
0, & \text { otherwise } \\
-\theta\left(-D_{p}\left(\psi_{i j}, \psi_{k j}\right)\right)^{\beta}, & \psi_{k j} \succ \psi_{i j}
\end{array}\right.
\end{aligned}
$$

\section{Step 3.4. Obtain the preference index}

According to the PROMETHEE II method, the preference index can be calculated as:

$$
\rho\left(\psi_{i}, \psi_{k}\right)=\sum_{1 \leq j \leq m, \psi_{i} \neq \psi_{k}} \varpi_{j} \cdot v_{j}^{1}\left(\psi_{i}, \psi_{k}\right)(i=1,2, \ldots, \mathrm{n} ; k=1,2, \ldots, n) ;
$$




$$
\rho\left(\psi_{k}, \psi_{i}\right)=\sum_{1 \leq j \leq m, \psi_{k} \neq \psi_{i}} \varpi_{j} \cdot v_{j}^{2}\left(\psi_{i}, \psi_{k}\right) \quad(i=1,2, \ldots, \mathrm{n} ; k=1,2, \ldots, n),
$$

where $\varpi_{j}$ denotes the weight assigned to criterion $c_{j}$.

\section{Step 3.5. Obtain the positive and negative flows}

The positive flow $\tau^{+}\left(\psi_{i}\right)$ and negative flow $\tau^{-}\left(\psi_{i}\right)$ can be defined respectively as:

$$
\begin{aligned}
& \tau^{+}\left(\psi_{i}\right)=\frac{1}{n-1} \sum_{\psi_{k} \neq \psi_{i}} \rho\left(\psi_{i}, \psi_{k}\right)(i=1,2, \ldots, \mathrm{n} ; k=1,2, \ldots, n) ; \\
& \tau^{-}\left(\psi_{i}\right)=\frac{1}{n-1} \sum_{\psi_{i} \neq \psi_{k}} \rho\left(\psi_{k}, \psi_{i}\right)(i=1,2, \ldots, \mathrm{n} ; k=1,2, \ldots, n) .
\end{aligned}
$$

Here $n$ is the number of alternatives.

\section{Step 3.6. Obtain the net flow}

From Eq. (9), the net flow $\tau\left(\psi_{i}\right)$ for alternative $\psi_{i}$ can be calculated.

\section{Step 3.7. Rank the alternatives}

From the net outranking flow, the alternatives can be ranked in a descending order. The larger the value of $\tau\left(\psi_{i}\right)$, the better the alternative $\psi_{i}$ should be.

\section{Case study: results and analysis}

Hubei Province is one of the most important industrial bases and tourism destinations in China. Recently, tourism has become an important strategic pillar industry of Hubei, and plays a key role in economic and social development. However, the environmental impact of tourism attractions, including positive and negative impact, is increasingly prominent in Hubei, which attracts wide attention of the public. For example, some residents think that environment is not as healthy and safe as before; while others think that more diversified tourist types in the medium-to-long term can yield greater regional economic benefits. In recent years, the government and experts have proposed some objectives for environmental protection and sustainable development of tourism. However, the environment is affected by the number of tourists, ecology, climate and society, and so on. Moreover, the impact degree also has bigger subjectivity. At present, in order to provide the relevant suggestions for the environmental protection and the tourism sustainable development, Hubei needs to implement EIA of tourism attractions as soon as possible.

Famous tourism attractions in Hubei include the Enshi Grand Canyon, the Yellow Crane Tower, the Three Gorges Dam, the East Lake, the Wudang Mountain, and the Shennongjia Nature Reserve. For the healthy and sustainable development of tourism industry in Hubei, the tourism department should assess the environmental impacts of these six famous tourism attractions. The Enshi Grand Canyon, i.e., $\psi_{1}$, lies in Tujia Miao Autonomous Prefecture of Enshi, and there are precipitous mountain peaks, large cliffs, and high waterfalls in the anyon. The Qingjiang River, at the bottom of the canyon, is crystal clear and invigorating. 
The Yellow Crane Tower, i.e., $\psi_{2}$, located at the top of Snake Mountain on the south bank of the Yangze River Bridge in Wuhan, is one of China's most famous towers. It has been known since ancient times as "the first floor of the World Mountains" and "the most beautiful attraction in the world". The Three Gorges Dam, i.e., $\psi_{3}$, located in Yichang, Hubei Province, was officially opened to the public in 1997. It offers a comprehensive engineering culture and water conservancy culture, and provides multi-functional services for tourists in the forms of sightseeing, science, education, leisure and entertainment. The East Lake, i.e., $\psi_{4}$, located in Wuhan, Hubei Province, has a large natural lake as its core, with a lakeside and mountain color. It is the largest tourism attraction in central of Wuhan. Wudang Mountain, i.e., $\psi_{5}$, known as Taihe Mountain in ancient times, is located in Shiyan, Hubei Province. It is the birthplace of Taoist famous mountains and Wudang Wushu. Wudang Wushu is an important school of Chinese Wushu. Large Taoist architectures can be seen everywhere on Wudang Mountain. Shennongjia Nature Reserve, i.e., $\psi_{6}$, was established in 1982, and it is the first "Treble King" heritage site of China, which has been entered into the UNESCO Man and Biosphere Nature Reserves list, the World Geoparks list and the World Heritage Sites list. These six tourism attractions have characteristics of unique landscape resources, rich tourism resources, abundant folk culture, and superior transport infrastructure. Based on the criteria index system for EIA of tourism attractions described in Subsection 2.1, related experts were consulted to provide assessment values for these six tourism attractions, using the three criteria and eight sub-criteria.

\subsection{Results}

In this subsection, a procedure to assess and choose the most optimal tourism attraction(s) by using the improved AHP method and the picture fuzzy PROMETHEE II method is provided.

\section{Phase I. Construct criteria for EIA of tourism attractions}

In this phase, twenty experts are consulted, including environmental experts, academicians, and tourism environment associates. Based on their suggestions and relevant literature reviews, three criteria and eight sub-criteria are identified to assess the environment impact of tourism attractions as presented in Table 1.

\section{Phase II. Determine the weights of criteria using the improved AHP method}

Step 2.1. Construct the analytical hierarchy structure

According to the criteria in Table 1, the analytical hierarchy structure for EIA of tourism attractions is constructed, as presented in Figure 3.

\section{Step 2.2. Determine fuzzy pair-wise comparison matrices}

The experts invited in Phase I are consulted to assess all criteria and sub-criteria. These selected experts have professional knowledge about EIA, and provide information about the degree of importance of each sub-criterion based on the fuzzy linguistic scales in Table 2, to 


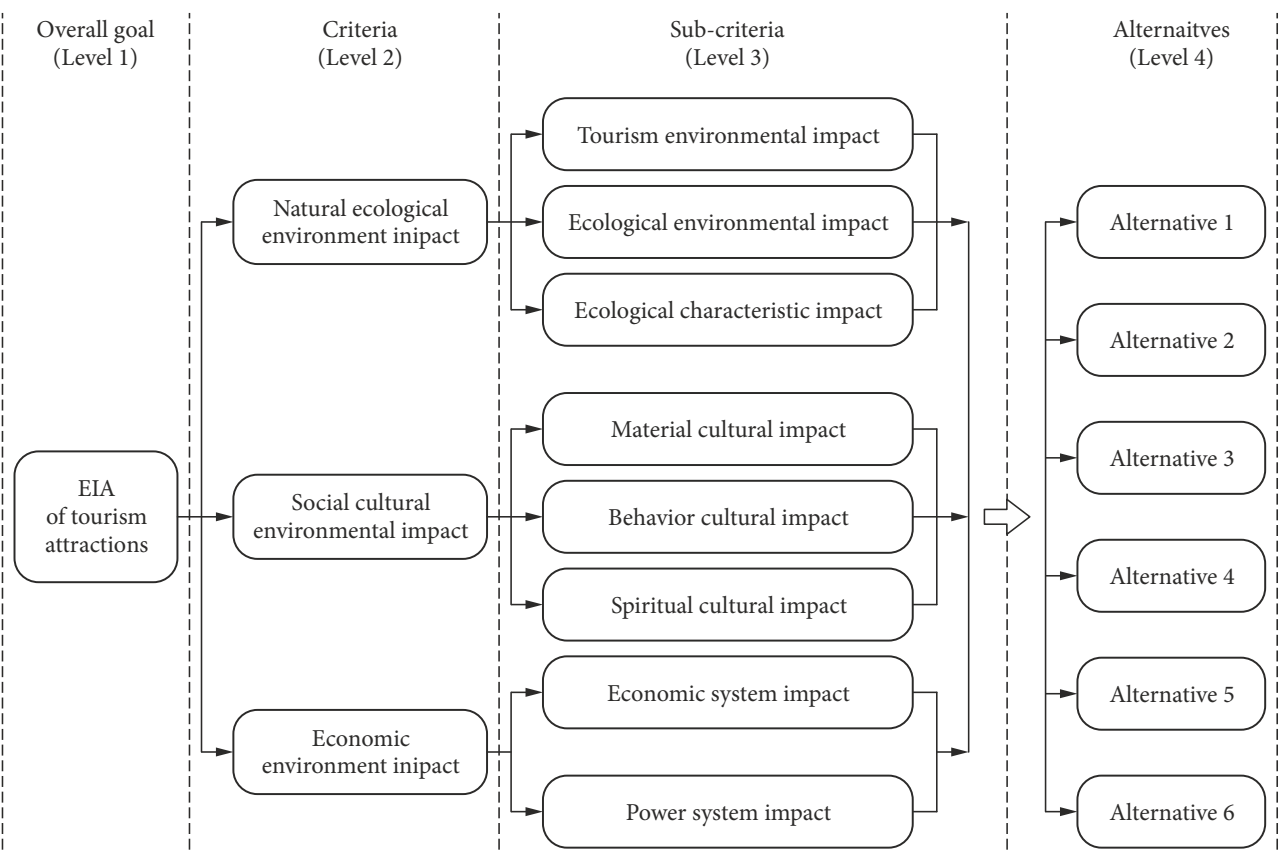

Figure 3. Analytical hierarchy structure for EIA of tourism attractions

construct the comparison matrices. The corresponding data are then analyzed using Super Decision Software based on the expert mean assessment method (Zhang et al., 2018a) (Figure 2). Since the consistency ratios of all pair-wise comparison matrices do not exceed 0.1 , the pair-wise comparison matrices are valid.

\section{Step 2.3. Determine the weights of criteria}

From the results in Step 2.2, the weight distributions of criteria are obtained by using the Super Decision Software, as presented in Table 4.

Table 4. Weight distributions of criteria for EIA of tourism attractions

\begin{tabular}{|c|c|c|c|c|}
\hline Criteria & Sub-criteria & $\begin{array}{c}\text { Criteria } \\
\text { weight }\end{array}$ & $\begin{array}{c}\text { Relative } \\
\text { weight }\end{array}$ & $\begin{array}{l}\text { Comprehensive } \\
\text { weight }\end{array}$ \\
\hline \multirow{3}{*}{$\begin{array}{l}\text { (C1) Natural } \\
\text { ecological } \\
\text { environmental } \\
\text { impact }\end{array}$} & (C11) Tourism environmental impact & 0.090 & 0.282 & \multirow{3}{*}{0.319} \\
\hline & (C12) Ecological environmental impact & 0.104 & 0.326 & \\
\hline & (C13) Ecological characteristic impact & 0.125 & 0.392 & \\
\hline \multirow{3}{*}{$\begin{array}{l}\text { (C2) Social cultural } \\
\text { environmental } \\
\text { impact }\end{array}$} & (C21) Material cultural impact & 0.177 & 0.439 & \multirow{3}{*}{0.403} \\
\hline & (C22) Behavior cultural impact & 0.102 & 0.253 & \\
\hline & (C23) Spiritual cultural impact & 0.124 & 0.308 & \\
\hline \multirow{2}{*}{$\begin{array}{l}\text { (C3) Economic } \\
\text { environmental } \\
\text { impact }\end{array}$} & (C31) Economic system impact & 0.122 & 0.439 & \multirow{2}{*}{0.278} \\
\hline & (C32) Power system impact & 0.156 & 0.561 & \\
\hline
\end{tabular}

Thus, the weight vector of criteria for EIA of tourism attractions is $\varpi=(0.319,0.403,0.278)^{T}$. 


\section{Phase III. The extended picture fuzzy PROMETHEE II method}

\section{Steps 3.1-3.2. Establish and normalize the decision matrix}

According to their knowledge and experience, the experts invited in Phase I provides assessment values in the form of PFNs $\psi_{i j}(i=1,2,3,4,5,6 ; j=1,2,3)$ for each tourism attraction based on three criteria, i.e., natural ecological environmental impact $c_{1}$, social cultural environmental impact $c_{2}$ and economic environmental impact $c_{3}$.

For instance, the criteria for each tourism attraction can be assessed by experts at three levels: high, medium and low. Moreover, if the expert's knowledge is limited, he/she can refuse to provide an assessment value. In other words, the options of high, medium, low and refusal in the assessment process correspond to four parameters of PFNs, namely, positive, neutral, negative and refusal memberships, respectively. In order to assure the precision and effectiveness of the assessment information, no corresponding information is provided during the assessment process, and the experts are not allowed to communicate with each other. Moreover, since the importance of the invited experts is the same, the final decision matrix, as presented in Table 5, can be obtained by averaging each membership degree in the PFNs provided by them.

Table 5. The picture fuzzy decision matrix

\begin{tabular}{|c|c|c|c|}
\hline & $c_{1}$ & $c_{2}$ & $c_{3}$ \\
\hline$\psi_{1}$ & $(0.65,0.13,0.21)$ & $(0.73,0.03,0.15)$ & $(0.64,0.11,0.15)$ \\
\hline$\psi_{2}$ & $(0.69,0.11,0.19)$ & $(0.65,0.07,0.23)$ & $(0.76,0.09,0.02)$ \\
\hline$\psi_{3}$ & $(0.77,0.05,0.14)$ & $(0.62,0.12,0.17)$ & $(0.66,0.08,0.21)$ \\
\hline$\psi_{4}$ & $(0.65,0.11,0.21)$ & $(0.69,0.10,0.13)$ & $(0.65,0.14,0.2)$ \\
\hline$\psi_{5}$ & $(0.73,0.06,0.19)$ & $(0.72,0.07,0.12)$ & $(0.60,0.13,0.20)$ \\
\hline$\psi_{6}$ & $(0.67,0.13,0.10)$ & $(0.74,0.06,0.15)$ & $(0.64,0.10,0.12)$ \\
\hline
\end{tabular}

Moreover, because all criteria are benefit types, and have the same measurement units, there is no need for normalization, i.e., $\tilde{R}=\left(\tilde{\psi}_{i j}\right)_{6 \times 3}=\left(\psi_{i j}\right)_{6 \times 3}$.

\section{Step 3.3. Find the preference function}

Based on Eqs. (10) and (11), it is assumed that $\alpha=\beta=0.88, \theta=2.25$ (Tversky \& Kahneman, 1992). $D_{p}\left(\psi_{i j}, \psi_{k j}\right)$ can be calculated by using the picture fuzzy Euclidean distance with $\lambda=2$, i.e., Eq. (4). Then, the prospect matrices can be obtained as shown in Tables 6 and 7 , respectively. 
Table 6. The prospect value by using the function $v_{j}^{1}\left(\psi_{i}, \psi_{k}\right)$

\begin{tabular}{|c|c|c|c|c|c|c|c|}
\hline$v_{j}^{1}\left(\psi_{1}, \psi_{k}\right)$ & 0 & 0 & 0 & $v_{j}^{1}\left(\psi_{4}, \psi_{k}\right)$ & 0.0078 & 0 & 0 \\
\hline & 0 & 0.0497 & 0 & & 0 & 0.0390 & 0 \\
\hline & 0 & 0.0619 & 0.0250 & & 0 & 0.0491 & 0 \\
\hline & 0 & 0.0321 & 0.0206 & & 0 & 0 & 0 \\
\hline & 0 & 0.0183 & 0.0285 & & 0 & 0 & 0.0283 \\
\hline & 0 & 0.0126 & 0 & & 0 & 0 & 0 \\
\hline$v_{j}^{1}\left(\psi_{2}, \psi_{k}\right)$ & 0.0248 & 0 & 0.0706 & $v_{j}^{1}\left(\psi_{5}, \psi_{k}\right)$ & 0.0248 & 0 & 0 \\
\hline & 0 & 0 & 0 & & 0.0278 & 0.0493 & 0 \\
\hline & 0 & 0.0302 & 0.0735 & & 0 & 0.0555 & 0 \\
\hline & 0.0240 & 0 & 0.0759 & & 0.0453 & 0.0207 & 0 \\
\hline & 0 & 0 & 0.0922 & & 0 & 0 & 0 \\
\hline & 0 & 0 & 0.0668 & & 0.0468 & 0.0164 & 0 \\
\hline$v_{j}^{1}\left(\psi_{3}, \psi_{k}\right)$ & 0.0674 & 0 & 0 & $v_{j}^{1}\left(\psi_{6}, \psi_{k}\right)$ & 0.0363 & 0 & 0.0116 \\
\hline & 0.0481 & 0 & 0 & & 0.0316 & 0.0525 & 0 \\
\hline & 0 & 0 & 0 & & 0 & 0.0633 & 0.0316 \\
\hline & 0.0659 & 0 & 0.0214 & & 0.0367 & 0.0313 & 0.0295 \\
\hline & 0.0280 & 0 & 0.0364 & & 0 & 0 & 0.0348 \\
\hline & 0.0575 & 0 & 0 & & 0 & 0 & 0 \\
\hline
\end{tabular}

Table 7. The prospect value by using the function $v_{j}^{2}\left(\psi_{i}, \psi_{k}\right)$

\begin{tabular}{|c|c|c|c|c|c|c|c|}
\hline$v_{j}^{2}\left(\psi_{1}, \psi_{k}\right)$ & 0 & 0 & 0 & $v_{j}^{2}\left(\psi_{4}, \psi_{k}\right)$ & 0 & 0.0672 & 0.0431 \\
\hline & 0.0518 & 0 & 0.1477 & & 0.0502 & 0. & 0.1587 \\
\hline & 0.1411 & 0 & 0 & & 0.1378 & 0 & 0.0447 \\
\hline & 0.0162 & 0 & 0 & & 0 & 0 & 0 \\
\hline & 0.0992 & 0 & 0 & & 0.0948 & 0.0432 & 0 \\
\hline & 0.0759 & 0 & 0.0243 & & 0.0768 & 0.0654 & 0.0616 \\
\hline$v_{j}^{2}\left(\psi_{2}, \psi_{k}\right)$ & 0 & 0.1039 & 0 & $v_{j}^{2}\left(\psi_{5}, \psi_{k}\right)$ & 0 & 0.0382 & 0.0596 \\
\hline & 0 & 0 & 0 & & 0 & 0 & 0.1928 \\
\hline & 0.1007 & 0 & 0 & & 0.0586 & 0 & 0.0761 \\
\hline & 0 & 0.0816 & 0 & & 0 & 0 & 0.0593 \\
\hline & 0.0583 & 0.1032 & 0 & & 0 & 0 & 0 \\
\hline & 0.0660 & 0.1098 & 0 & & 0 & 0 & 0.0728 \\
\hline & 0 & 0.1294 & 0.0522 & $v_{j}^{2}\left(\psi_{6}, \psi_{k}\right)$ & 0 & 0.0263 & 0 \\
\hline & 0 & 0.0632 & 0.1537 & & 0 & 0 & 0.1397 \\
\hline & 0 & 0 & 0 & & 0.1204 & 0 & 0 \\
\hline & 0 & 0.0838 & 0 & & 0 & 0 & 0 \\
\hline & 0 & 0.1161 & 0 & & 0.0980 & 0.0343 & 0 \\
\hline & 0 & 0.1324 & 0.0660 & & 0 & 0 & 0 \\
\hline
\end{tabular}




\section{Step 3.4. Obtain the preference index}

From Eqs (12) and (13), the corresponding preference index matrix can be obtained in Table 8.

Table 8. The preference index matrix

\begin{tabular}{|c|c|c|c|c|c|c|}
\hline$\rho\left(\psi_{i}, \psi_{k}\right)$ & - & 0.0200 & 0.0319 & 0.0187 & 0.0153 & 0.0051 \\
\hline & 0.0275 & - & 0.0326 & 0.0287 & 0.0256 & 0.0186 \\
\hline & 0.0215 & 0.0153 & - & 0.0270 & 0.0190 & 0.0184 \\
\hline & 0.0025 & 0.0157 & 0.0162 & - & 0.0079 & 0 \\
\hline & 0.0151 & 0.0288 & 0.0224 & 0.0228 & - & 0.0216 \\
\hline & 0.0148 & 0.0312 & 0.0343 & 0.0325 & 0.0097 & - \\
\hline$\rho\left(\psi_{k}, \psi_{i}\right)$ & - & 0.0576 & 0.0450 & 0.0052 & 0.0317 & 0.0309 \\
\hline & 0.0419 & - & 0.0321 & 0.0329 & 0.0602 & 0.0653 \\
\hline & 0.0667 & 0.0682 & - & 0.0338 & 0.0468 & 0.0717 \\
\hline & 0.0391 & 0.0601 & 0.0564 & - & 0.0477 & 0.0680 \\
\hline & 0.0320 & 0.0536 & 0.0398 & 0.0165 & - & 0.0202 \\
\hline & 0.0106 & 0.0388 & 0.0384 & 0 & 0.0451 & - \\
\hline
\end{tabular}

Step 3.5. Obtain the positive and negative flows

From Eqs (14) and (15), the positive flow and negative flow are obtained as shown in Tables 9 and 10, respectively.

Table 9. The positive flow

\begin{tabular}{|c|c|c|c|c|c|}
\hline$\tau^{+}\left(\psi_{1}\right)$ & $\tau^{+}\left(\psi_{2}\right)$ & $\tau^{+}\left(\psi_{3}\right)$ & $\tau^{+}\left(\psi_{4}\right)$ & $\tau^{+}\left(\psi_{5}\right)$ & $\tau^{+}\left(\psi_{6}\right)$ \\
\hline 0.0182 & 0.0266 & 0.0202 & 0.0084 & 0.0221 & 0.0245 \\
\hline
\end{tabular}

Table 10. The negative flow

\begin{tabular}{|c|c|c|c|c|c|}
\hline$\tau^{-}\left(\psi_{1}\right)$ & $\tau^{-}\left(\psi_{2}\right)$ & $\tau^{-}\left(\psi_{3}\right)$ & $\tau^{-}\left(\psi_{4}\right)$ & $\tau^{-}\left(\psi_{5}\right)$ & $\tau^{-}\left(\psi_{6}\right)$ \\
\hline 0.0341 & 0.0465 & 0.0574 & 0.0543 & 0.0324 & 0.0266 \\
\hline
\end{tabular}

\section{Step 3.6. Obtain the net flow}

From Eq. (9), the net flow of each tourism attraction can be obtained as shown in Table 11.

Table 11. The net flow

\begin{tabular}{|c|c|c|c|c|c|}
\hline$\tau\left(\psi_{1}\right)$ & $\tau\left(\psi_{2}\right)$ & $\tau\left(\psi_{3}\right)$ & $\tau\left(\psi_{4}\right)$ & $\tau\left(\psi_{5}\right)$ & $\tau\left(\psi_{6}\right)$ \\
\hline-0.0159 & -0.0199 & -0.0372 & -0.0458 & -0.0103 & -0.0021 \\
\hline
\end{tabular}




\section{Step 3.7. Rank the alternatives}

From Step 3.6, it can be seen that the net flows of the six tourism attractions follow the order of $\tau\left(\psi_{6}\right)>\tau\left(\psi_{5}\right)>\tau\left(\psi_{1}\right)>\tau\left(\psi_{2}\right)>\tau\left(\psi_{3}\right)>\tau\left(\psi_{4}\right)$. Thus, $\psi_{6} \succ \psi_{5} \succ \psi_{1} \succ \psi_{2} \succ \psi_{3} \succ \psi_{4}$ can be obtained. That is to say, the tourism environment of alternative $\psi_{2}$, i.e., Shennongjia Nature Reserve, is the optimal one, while that of alternative $\psi_{4}$, i.e., the East Lake, is the worst one.

\subsection{Sensitivity analysis}

In this sub-section, we investigated the influence of different parameters on the ranking of tourism attractions are investigated. For the first case, $\alpha=\beta=0.88$ are fixed, and the changes of parameters $\lambda$ and $\theta$, i.e., $0<\lambda \leq 10$ and $0<\theta \leq 5$, are considered; for the second, $\lambda=2$ and $\theta=2.25$ are fixed, and the changes of parameters $\alpha$ and $\beta$, i.e., $0<\alpha \leq 5$ and $0<\beta \leq 5$, are considered. The results of the two cases are shown in Figures 4-7.

For the first case, we can see that if $\lambda$ becomes greater while $\theta$ becomes smaller, then it is difficult to judge the positions for the Enshi Grand Canyon $\psi_{1}$ and the Yellow Crane Tower $\psi_{2}$. However, the changes of the two parameters do not influence the final decision, namely Shennongjia $\psi_{6}$ is always the optimal one. For the second case, based on the results presented in Figures 5-7, if $0<\alpha \leq 1$ and $0<\beta \leq 5$, then the position for Wudang Mountain $\psi_{5}$ cannot be judged, and the optimal one is the East Lake $\psi_{4}$; if $1<\alpha \leq 2$ and $0<\beta \leq 5$, then the optimal one is Shennongjia $\psi_{6}$. However, if $2<\alpha \leq 5$ and $2<\beta \leq 5$, then the final ranking cannot be obtained.

Apparently, different values of the two parameters $\alpha$ and $\beta$ have great influences on the final ranking. Generally speaking, decision-makers can determine the values of parameters according to their preferences. However, the values of the three parameters, i.e., $\alpha=\beta=0.88$ and $\theta=2.25$, in the PROMETHEE II method are the optimal values by using experimental

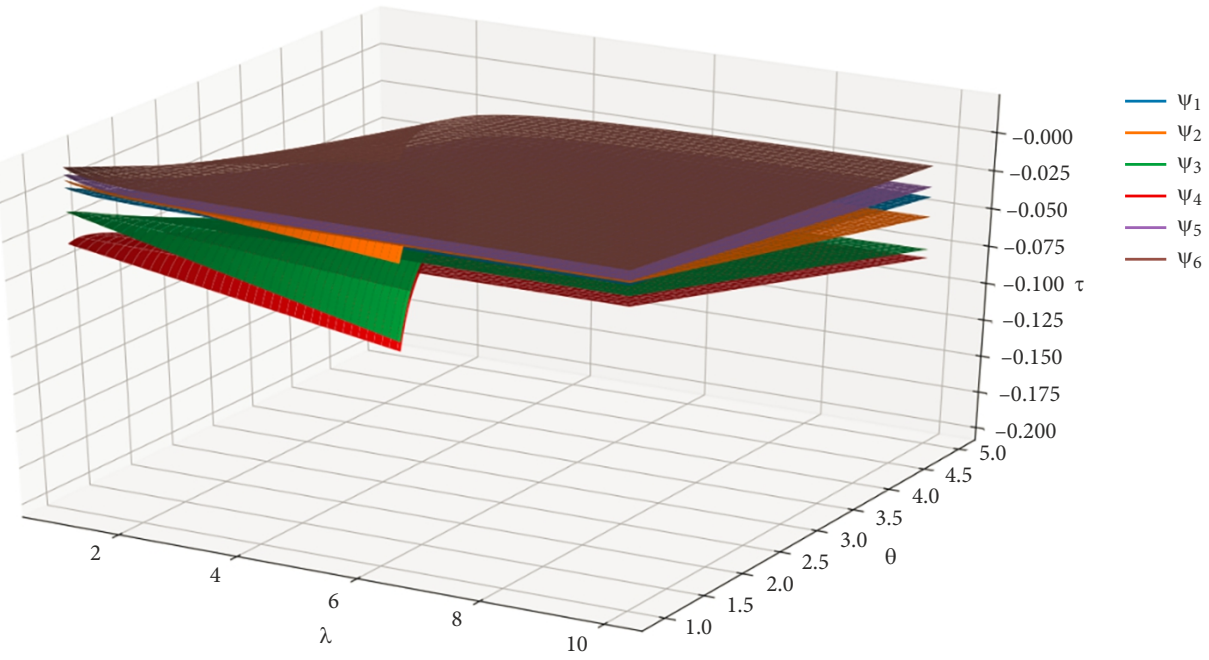

Figure 4. Sensitivity analysis with $\alpha=\beta=0.88,0<\theta \leq 5$ and $0<\lambda \leq 10$ 
analysis (Tversky \& Kahneman, 1992). Moreover, since the membership degrees of PFNs are between 0 and 1 , the differences between these elements by using the picture fuzzy distance are very small. Therefore, if $\lambda$ is too large, the differences for the distances of PFNs will be not obvious.

\subsection{Comparative analysis}

To prove the feasibility, comparative analysis is conducted by using existing picture fuzzy decision-making methods, i.e., Wei (2016), Wei (2017), L. Wang, Zhang, J. Q. Wang, \& Li, (2018b) and Wang et al. (2019b).

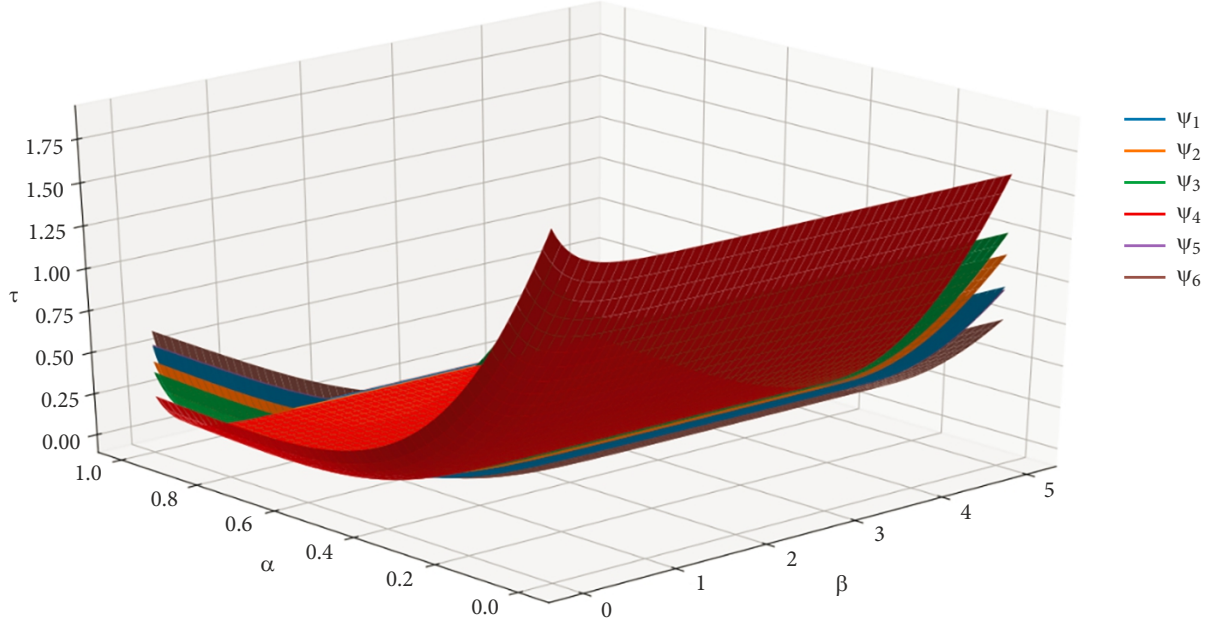

Figure 5. Sensitivity analysis with $\theta=2.25, \lambda=2,0<\alpha \leq 1$ and $0<\beta \leq 5$

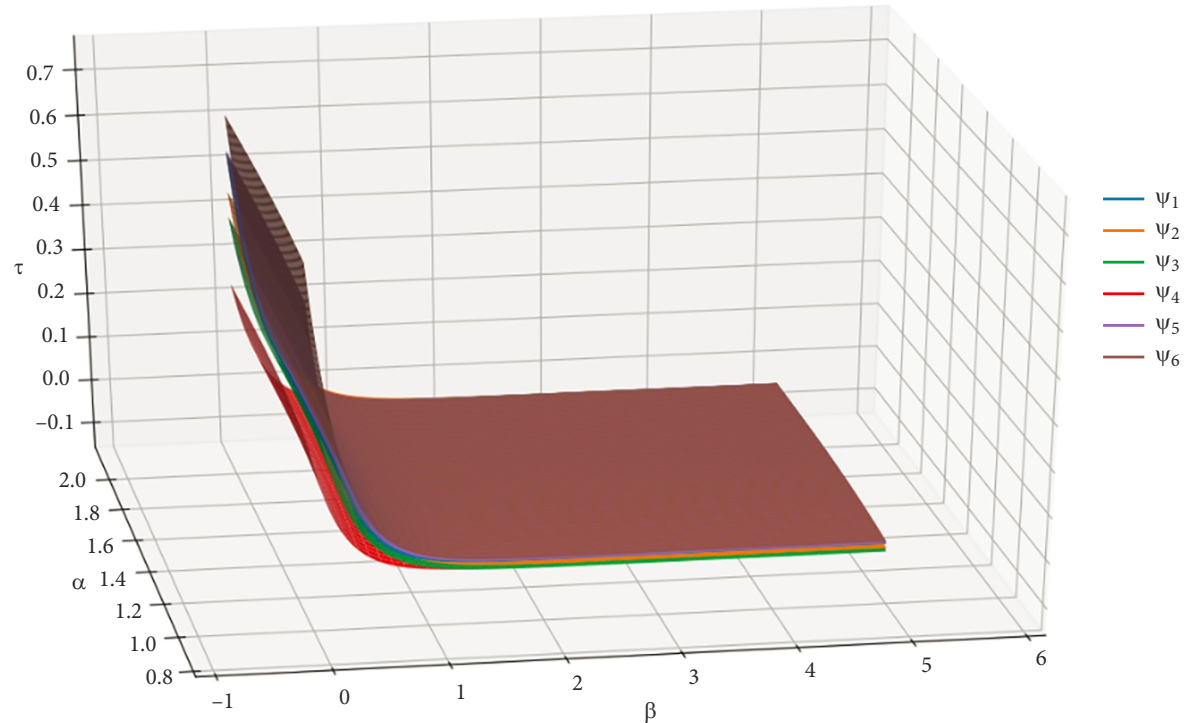

Figure 6. Sensitivity analysis with $\theta=2.25, \lambda=2,1<\alpha \leq 2$ and $0<\beta \leq 5$ 


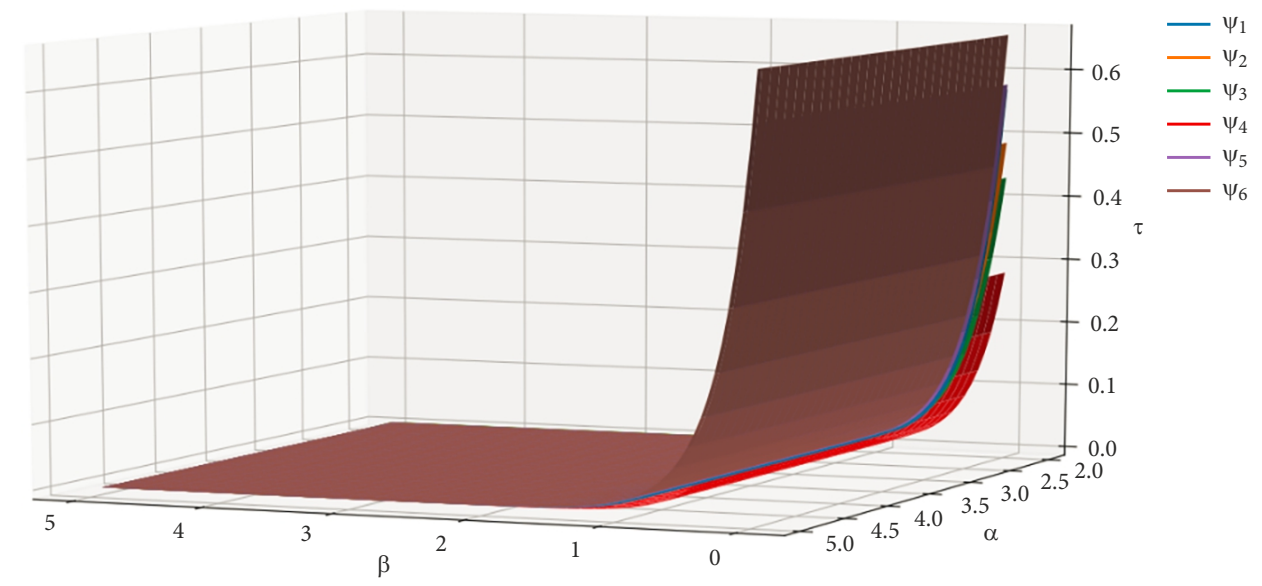

Figure 7. Sensitivity analysis with $\theta=2.25, \lambda=2,2<\alpha \leq 5$ and $0<\beta \leq 5$

The method proposed by Wei (2016), Wei (2017), and Wang et al. (2019b) cannot resolve special decision-making problems with unknown weight information. On account of this, the same case study is conducted where the weight is $\varpi=(0.313,0.195,0.217,0.275)^{T}$. Then, the cross-entropy between alternatives and the positive solution can be obtained and used to identify the final order of all alternatives in Wei (2016). For the method defined by Wang et al. (2018b), the corresponding weight can be obtained with $s=t=1$. Then the overall gain and loss of each alternative for all criteria with $\alpha=\beta=0.88$ and $\gamma=2.25$ can be obtained. For the methods in Wei (2017) and Wang et al. (2019b), the aggregated assessment value of each alternative can be obtained by using the weighted average operator and weighted Muirhead mean operator respectively. These above mentioned methods and the developed method are respectively used to address the same case, and the results are presented in Table 12.

Table 12. Comparative analysis results

\begin{tabular}{|l|l|l|}
\hline \multicolumn{1}{|c|}{ Methods } & \multicolumn{1}{c|}{ Methods } & \multicolumn{1}{c|}{ Ranking } \\
\hline Wei (2016) & Cross-entropy & $\psi_{6} \succ \psi_{5} \succ \psi_{1} \succ \psi_{2} \succ \psi_{3} \succ \psi_{4}$ \\
\hline Wei (2017) & Average operator & $\psi_{5} \succ \psi_{6} \succ \psi_{1} \succ \psi_{2} \succ \psi_{4} \succ \psi_{3}$ \\
\hline Wang et al. (2018b) & VIKOR & $\psi_{6} \succ \psi_{5} \succ \psi_{2} \succ \psi_{1} \succ \psi_{3} \succ \psi_{4}$ \\
\hline Wang et al. (2019b) & Muirhead mean operator & $\psi_{5} \succ \psi_{6} \succ \psi_{1} \succ \psi_{2} \succ \psi_{4} \succ \psi_{3}$ \\
\hline Proposed method & AHP and PROMETHEE II & $\psi_{6} \succ \psi_{5} \succ \psi_{1} \succ \psi_{2} \succ \psi_{3} \succ \psi_{4}$ \\
\hline
\end{tabular}

From Table 11, it can be seen that the final results by using the proposed method are same with that of method in Wei (2016), and are different from that of the methods proposed by Wei (2017) and Wang et al. (2019b). Moreover, although the ranking of the Enshi Grand Canyon $\psi_{1}$ and the Yellow Crane Tower $\psi_{2}$ changes when Wang et al's method (2018b) is used, the optimal alternative is always Shennongjia $\psi_{6}$. 


\section{Findings and discussion}

In this paper, an integrated decision-making method by using the improved AHP and the picture fuzzy PROMETHEE II method is developed to handle EIA of tourism attractions. This method can effectively handle the uncertainty information and improve the precision of decision-making. At the same time, the sensitivity analysis and comparative analysis are investigated by using the existing methods to verify the feasibility of the developed method,. The results indicate that the environment of Shennongjia Nature Reserve $\psi_{6}$ is the best.

From the above analysis, some findings are concluded in the following. First, the methods proposed in Wei (2016), Wei (2017), and Wang et al. (2019b) are constructed based on the strict rationality of decision-makers whereas the developed method considers the psychological behavior of decision-makers, which is more applicable to actual decision-making problems. Second, the picture fuzzy aggregation operators defined by Wei (2017) and Wang et al. (2019b) involve unreasonable operation rules (Zhang, Wang, \& Hu, 2018b) whereas the developed method can avoid this shortcoming. Third, the developed method can offer a flexible way to effectively deal with special decision-making problems with unknown weight information. Moreover, the expert mean assessment method is used to improve traditional AHP method, which can avoid human errors and obtain the most important weights of criteria in the assessment of environmental impact of tourism attractions. Therefore, the final ranking results by using the developed method are more credible.

\section{Implications}

\subsection{Research implications}

The main differences between the developed approach and other existing methods lie in that it integrates the improved AHP method and the picture fuzzy PROMETHEE II method, and can better describe uncertainty information with a positive, neutral, negative, and refusal degrees by PFNs.

This study mainly develops an integrated decision-making method for the EIA problem of tourism attractions, and has the following research implications. First, based on literature review, this study constructs an assessment index system of EIA consisting of natural ecological environmental impact, social cultural environmental impact and economic environmental impact criteria, as well as eight sub-criteria. Second, some research has presented several methods about EIA of tourism attractions but cannot consider the psychological behavior of decision-makers. Third, the picture fuzzy aggregation operators have their corresponding drawbacks as discussed in Zhang et al. (2018b), whereas the proposed method by using the picture fuzzy distance measure and the prospect theory is an efficient way for dealing with such problems, with an improved decision-making precision.

\subsection{Practical implications}

Since the cultural background and tourism preferences of different countries are diverse, most existing methods cannot meet the needs of all governments or management depart- 
ments. This study, which discusses the EIA problem of main tourism attractions in China, has certain practical implications.

First, EIA plays a key role in the sustainable development of tourism. The assessment index system in this study can highlight the "sustainable" characteristics and meet the requirements of new economic environment in China (Chang et al., 2018). Second, the improved AHP method is utilized to obtain the weight information, which can avoid spiteful assessment by decision-makers and improve the objectivity of the assessment process. Third, the assessment results by using the developed method are consistent with the actual situation. To be specific, Shennongjia Nature Reserve $\psi_{6}$ performs outstandingly in environmental protection, while the East Lake $\psi_{4}$ is the worst one. Moreover, Shennongjia Nature Reserve is a natural ecological tourism destination with the well-preserved ecological environment, forest ecosystem and biological diversity. There are extremely rich and precious natural and cultural landscapes, and the tourism environment is pleasant and graceful because of its unique natural environment and cultural history (Chang et al., 2018). According to the assessment results, the Yellow Crane Tower $\psi_{2}$ is in the fourth position. However, both the Yellow Crane Tower and the East Lake are located in the center of Wuhan, and the tourism resources and service facilities are always not enough to meet the tourists, which brings great negative impact on the environment. Hence, it is necessary to continuously improve tourism service facilities and basic public facilities, enhance the carrying capacity and quality of tourism attractions by tourist entertainment, strictly control the incoming pollution, and strongly protect the diversity of resources in the tourism attractions. The Three Gorges Dam $\psi_{3}$ is in the fifth position, and it is China's largest engineering project. Since the main purpose of the Three Gorges Dam is hydroelectric generation, the government should implement the project construction on the basis of sustainable development, and take various effective measures to actively protect the natural ecological environment. Moreover, the Enshi Grand Canyon $\psi_{1}$, the Yellow Crane Tower $\psi_{2}$, the Wudang Mountain $\psi_{5}$, and the Shennongia Nature Reserve $\psi_{6}$ should construct their own cultural tourism brands with distinguishing features, protect natural ecological environment and retain the traditional social culture to achieve the goal of sustainable development.

Therefore, the developed method is suitable for dealing with the EIA problem of tourism attractions, which can provide valuable references for the sustainable development of tourism and thus reduce environmental damages.

\section{Conclusions}

In summary, an integrated decision-making method that combines the improved AHP method and the picture fuzzy PROMETHEE II method is developed to handle the EIA problem of tourism attractions. The improved AHP method is utilized to obtain the weight information for EIA of tourism attractions, and then the extended picture fuzzy PROMETHEE II method is used to assess tourism attractions. The contributions for this study are concluded as follows. First, EIA of tourism attractions always involves incomplete, uncertain or imprecise information, while the introduction of PFNs in this study can precisely describe decision-makers' preference information when there is uncertain or incomplete information 
about EIA of tourism attractions. Second, the improved AHP method based on expert mean assessment is utilized to obtain the importance of different criteria in the decision-making process of EIA of tourism attractions, which can reduce the errors caused by human factors. Finally, an extended picture fuzzy PROMETHEE II method is proposed to assess tourism attractions, which consider the decision-makers' psychological behavior. Additionally, an illustrative example is presented to prove the feasibility and effectiveness of the proposed approach for EIA of tourism attractions. Sensitivity analysis and comparative analysis confirm that the developed method is credible and efficient.

There are two main limitations in this study. First, only twenty experts are invited to determine the weight of criteria for EIA of tourism attractions, which may have an impact on the final decision-making results. Second, the developed method cannot consider the relationship between criteria. However, not all of criteria are independent in some real decision-making problems. In further research, we will continue to study the corresponding decision-making methods involving a large number of decision-makers and the interrelationship among multiple criteria simultaneously.

\section{Acknowledgements}

The authors are grateful for the comments provided by editors and reviewers to improve this paper. This work is supported by the National Natural Science Foundation of China (Nos. 71701065, 51475410, 51875503 and 71571193) and the Postdoctoral Science Foundation of China (No. 2017M610511).

\section{Disclosure statement}

The authors declare that there is no conflict of interest regarding the publication of this paper.

\section{References}

Atanassov, K. (1986). Intuitionistic fuzzy sets. Fuzzy Sets and Systems, 20, 87-96. https://doi.org/10.1016/S0165-0114(86)80034-3

Ayag, Z., \& Ozdemir, R. G. (2009). A hybrid approach to concept selection through fuzzy analytic newwork process. Computers \& Industrial Engineering, 56(1), 368-379. https://doi.org/10.1016/j.cie.2008.06.011

Brans, J. P. (1982). Lingenierie de la decision, elaboration dinstruments daide a la decision, methode PROMETHEE. In R. Nadeau, \& M. Landry (Eds.), Laide a la decision: nature, instrument set perspectives davenir (pp. 183-214). Qu ebec, Canada: Presses de Universite Laval.

Cuong, B. C., \& Kreinovich, V. (2013). Picture fuzzy sets-a new concept for computational intelligence problems. In Proceedings of $3 \mathrm{rd}$ World Congress on Information and Communication Technologies (WICT) (pp. 1-6). https://doi.org/10.1109/WICT.2013.7113099

Cegan, J. C., Filion, A. M., Keisler, J. M., \& Linkov, I. (2017). Trends and applications of multi-criteria decision analysis in environmental sciences: literature review. Environment Systems \& Decisions, 37, 123-133. https://doi.org/10.1007/s10669-017-9642-9 
Çalik, A., Yapici Pehlivan, N., \& Kahraman, C. (2018). An integrated fuzzy AHP/DEA approach for performance evaluation of territorial units in Turkey. Technological and Economic Development of Economy, 24(4), 1280-1302. https://doi.org/10.3846/20294913.2016.1230563

Cowburna, B., Moritza, C., Birrella, C., Grimsditcha, G., \& Abdulla, A. (2018). Can luxury and environmental sustainability co-exist? Assessing the environmental impact of resort tourism on coral reefs in the Maldives. Ocean and Coastal Management, 158, 120-127. https://doi.org/10.1016/j.ocecoaman.2018.03.025

Canteiro, M., Córdova-Tapia, F., \& Brazeiro, A. (2018). Tourism impact assessment: a tool to evaluate the environmental impacts of touristic activities in Natural Protected Areas. Tourism Management Perspectives, 28, 220-227. https://doi.org/10.1016/j.tmp.2018.09.007

Chang, S., Wang, W., Wu, J., Sun, Y., \& Hu, R. (2018). Environmental impact assessment follow-up for projects in China: Institution and practice. Environmental Impact Assessment Review, 73, 7-19. https://doi.org/10.1016/j.eiar.2018.06.005

Duan, H., Su, Z. X., Xu, X., \& Ran, Q. (2008). To evaluate eco-environmental quality of the co-environmental quality of the eco-agricultural tourism with RS \& GIS theory cased on Mulao country in Nanchong. Yunnan Geographic Environment Research, 20(5), 6-11.

Emrouznejad, A., \& Marra, M. (2017). The state of the art development of AHP (1979-2017): a literature review with a social network analysis. International Journal of Production Research, 7543, 1-23. https://doi.org/10.1080/00207543.2017.1334976

Elvan, D. O. (2018). Analysis of environmental impact assessment practices and legislation in Turkey. Environmental Science and Policy, 84, 1-6. https://doi.org/10.1016/j.envsci.2018.02.008

Gossling, S., Peeters, P., Ceron, J.-P., Dubois, G., Patterson, T., \& Richardson, R. B. (2005). The ecoefficiency of tourism. Ecological Economics, 54, 417-434.

https://doi.org/10.1016/j.ecolecon.2004.10.006

Geneletti, D., \& Dawa, D. (2009). Environmental impact assessment of mountain tourism in developing regions: a study in Ladakh, Indian Himalaya. Environmental Impact Assessment Review, 29, 229-242. https://doi.org/10.1016/j.eiar.2009.01.003

Greco, G., Cenciarelli, G. V., \& Allegrini, M. (2018). Tourism's impacts on the costs of municipal solid waste collection: evidence from Italy. Journal of Cleaner Production, 177, 62-68. https://doi.org/10.1016/j.jclepro.2017.12.179

Gunduz, M., \& Alfar, M. (2019). Integration of innovation through analytical hierarchy process (AHP) in project management and planning. Technological and Economic Development of Economy, 25(2), 258-276. https://doi.org/10.3846/tede.2019.8063

Kabir, G., \& Sumi, R. S. (2014). Power substation location selection using fuzzy analytic hierarchy process and PROMETHEE: A case study from Bangladesh. Energy, 72, 717-730. https://doi.org/10.1016/j.energy.2014.05.098

Khosravi, F., Jha-Thakur, U., \& Fischer, T. B. (2019). Evaluation of the environmental impact assessment system in Iran. Environmental Impact Assessment Review, 74, 63-72. https://doi.org/10.1016/j.eiar.2018.10.005

Luo, H. S. (2002). Environmental impact assessment of regional tourism. Journal of Yunnan Normal University, 22(3), 53-58.

Li, X. M., Zhang, J. S., \& Wang, F. F. (2005). EIA method and experience for ecological tourism projectCase study of Wuyishan great canyon forest ecological tourism district. Chinese Journal of Ecology, 24(9), 1110-1114.

Li, L. T., Xue, C. H., Ren, J., Zhang, G. L., \& Wang, Y. R. (2015). Comprehensive evaluation of influences of tourism activities on desert ecological environment of Ningxia based on the fuzzy theory. Tourism Research, 7(02), 45-51. 
Liu, P., \& Liu, W. (2018). Intuitionistic fuzzy interaction Maclaurin symmetric means and their application to multiple-attribute decision-making. Technological and Economic Development of Economy, 24(4), 1533-1559. https://doi.org/10.3846/tede.2018.3698

Michailidou, V. A., Vlachokostas, C., Moussiopoulos, N., \& Maleka, D. (2016). Life cycle thinking used for assessing the environmental impacts of tourism activity for a Greek tourism destination. Journal of Cleaner Production, 111, 499-510. https://doi.org/10.1016/j.jclepro.2015.09.099

MacNeill, T., \& Wozniak, D. (2018). The economic, social, and environmental impacts of cruise tourism. Tourism Management, 66, 387-404. https://doi.org/10.1016/j.tourman.2017.11.002

Ocampo-Melgara, A., Sagarisb, L., \& Gironás, J. (2019). Experiences of voluntary early participation in environmental impact assessments in Chilean mining. Environmental Impact Assessment Review, 74, 43-53. https://doi.org/10.1016/j.eiar.2018.09.004

Saaty, T. L. (1988). The analytic hierarchy process. New York: Pergamon. https://doi.org/10.1007/978-3-642-83555-1_5

Saaty, T. L. (1990). How to make a decision: the analytic hierarchy process. European Journal Operational Research, 48(1), 9-26. https://doi.org/10.1016/0377-2217(90)90057-I

Sennaroglu, B., \& Celebi, G. V. (2018). A military airport location selection by AHP integrated PROMETHEE and VIKOR methods. Transportation Research Part D, 59, 160-173. https://doi.org/10.1016/j.trd.2017.12.022

Salari, M., Rakhshandehroo, G. R., \& Nikoo, M. R. (2018). Degradation of ciprofloxacin antibiotic by homogeneous Fenton oxidation: hybrid AHP-PROMETHEE method, optimization, biodegradability improvement and identification of oxidized byproducts. Chemospher, 206, 157-167. https://doi.org/10.1016/j.chemosphere.2018.04.086

Su, M. M., Wall, G., Wang, Y., \& Jin, M. (2019). Livelihood sustainability in a rural tourism destination Hetu Town, Anhui Province, China. Tourism Management, 71, 272-281. https://doi.org/10.1016/j.tourman.2018.10.019

Tversky, A., \& Kahneman, D. (1992). Advances in prospect theory: cumulative representation of uncertainty. Journal of Risk \& Uncertainty, 5(4), 297-323. https://doi.org/10.1007/BF00122574

Tseng, M. L., Wu, K. J., Lee, C. H., Lim, K. M., Bui, T. D., \& Chen, C. C. (2018). Assessing sustainable tourism in Vietnam: a hierarchical structure approach. Journal of Cleaner Production, 195, 406-417. https://doi.org/10.1016/j.jclepro.2018.05.198

Thanvisitthpon, N. (2016). Urban environmental assessment and social impact assessment of tourism development policy: Thailand's Ayutthaya historical park. Tourism Management Perspectives, 18, 1-5. https://doi.org/10.1016/j.tmp.2016.01.006

Tian, C., Peng, J. J., Zhang, S., Zhang, W. Y., \& Wang, J. Q. (2019). Weighted picture fuzzy aggregation operators and their applications to multi-criteria decision-making problems. Computers \& Industrial Engineering, 137, 106037. https://doi.org/10.1016/j.cie.2019.106037

Vincke, Ph., \& Brans, J. P. (1985). A preference ranking organization method, the PROMETHEE method for MCDM. Management Science, 31, 641-656. https://doi.org/10.1287/mnsc.31.6.647

Wei, G. W. (2016). Picture fuzzy cross-entropy for multiple attribute decision making problems. Journal of Business Economics and Management, 17(4), 491-502. https://doi.org/10.3846/16111699.2016.1197147

Wei, G. (2017). Picture fuzzy aggregation operators and their application to multiple attribute decision making. Journal Intelligent \& Fuzzy Systems, 33, 713-724. https://doi.org/10.3233/JIFS-161798

Wang, L., Peng, J. J., \& Wang, J. Q. (2018a). A multi-criteria decision-making framework for risk ranking of energy performance contracting project under picture fuzzy environment. Journal of Cleaner Production, 191, 105-118. https://doi.org/10.1016/j.jclepro.2018.04.169 
Wang, L., Zhang, H. Y., Wang, J. Q., \& Li, L. (2018b). Picture fuzzy normalized projection-based VIKOR method for the risk evaluation of construction project. Applied Soft Computing, 64, 216-226. https://doi.org/10.1016/j.asoc.2017.12.014

Wang, L., Zhang, H. Y., Wang, J. Q., \& Wu, G. F. (2019a). Picture fuzzy multi-criteria group decisionmaking method to hotel building energy efficiency retrofit project selection. RAIRO-Operations Research. https://doi.org/10.1051/ro/2019004

Wang, R., Wang, J., Gao, H., \& Wei, G. (2019b). Methods for MADM with picture fuzzy Muirhead mean operators and their application for evaluating the financial investment risk. Symmetry, 11(1), 6. https://doi.org/10.3390/sym11010006

Wang, L., Wang, X. K., Peng, J. J., \& Wang, J. Q. (2020). The differences in hotel selection among various types of travellers: A comparative analysis with a useful bounded rationality behavioural decision support model. Tourism Management, 76, 103961. https://doi.org/10.1016/j.tourman.2019.103961

Ye, Y. P. \& Liu, L. J. (2000). A preliminary study on assessment indicator system of provincial ecoenvironmental quality in China. Research of Environmental Sciences, 13(3), 33-36.

Yin, D. L., \& Han, S. (2009). Environment impact appraise and protection of tourism area. Territory \& Natural Resources Study, 3, 61-62.

Yang, Y., Hu, J. H., Liu, Y. M., \& Chen, X. H. (2019). Alternative selection of end-of-life vehicle management in China: A group decision-making approach based on picture hesitant fuzzy measurements. Journal of Cleaner Production, 206, 631-645. https://doi.org/10.1016/j.jclepro.2018.09.188

Zhong, L., Deng, J., Song, Z., \& Ding, P. (2011). Research on environmental impacts of tourism in China: Progress and prospect. Journal of Environmental Management, 92(11), 2972-2983. https://doi.org/10.1016/j.jenvman.2011.07.011

Zhao, H. X., \& Zhang, X. Q. (2017). Building index system of tourism environmental impact assessment in the Desert Park based on intuitionistic fuzzy multiple attribute decision making method. Issues of Forestry Economics, 37(2), 55-60.

Zhang, Y. P., Wang, Y. H., \& Yang, N. (2018a). On the risk assessment method of balise system based on the ANP and fuzzy evidence theory. Journal of Safety and Environment, 18(2), 434-440.

Zhang, X. Y., Wang, J. Q., \& Hu, J. H. (2018b). On novel operational laws and aggregation operators of picture 2-tuple linguistic information for MCDM problems. International Journal of Fuzzy Systems, 20(3), 958-969. https://doi.org/10.1007/s40815-017-0441-8

Zhuang, Z. Y., Su, C. R., \& Chang, S. C. (2019). The effectiveness of IF-MADM (intuitionistic-fuzzy multi-attribute decision-making) for group decisions: methods and an empirical assessment for the selection of a senior centre. Technological and Economic Development of Economy, 25(2), 322-364. https://doi.org/10.3846/tede.2019.8399 